\title{
Review of: "CYP2J2 and EETs Protect Against Pulmonary Hypertension with Lung Ischemia- Reperfusion Injury In Vivo and In Vitro"
}

\author{
Theresa Aliwarga ${ }^{1}$ \\ 1 University of Washington
}

Potential competing interests: The author(s) declared that no potential competing interests exist.

The authors elucidate the protective effect of overexpression of CYP2J2 and addition of exogenous epoxyeicosatrienoic acids (EETs) in pulmonary arterial hypertension (PAH) with lung ischemia-reperfusion injury (LIRI). The positive features of the paper are the authors attempt to address the protective effect of EETs in a complex disease state and the use of both in vitro and in vivo model system to support their hypothesis. The lack of details in the study design is a drawback to the paper. Besides CYP2J2, CYP2C isozymes are the main epoxygenases in human (Xu, Zhang, Wang, 2011) and CYP2Cs isozymes are expressed in higher level than CYP2J2 in human endothelial cells ${ }^{[1]}$. It is not clear the basal expression levels of these epoxygenases in the human pulmonary artery endothelial cells (HPAECs). The addition of exogenous 11,12-EET to the HPAECs is very interesting. EETs are like other lipid molecules will bind readily to serum proteins. It is of interest to perform the study in a serum-free medium. In addition, this treatment with 11,12-EET cannot fully support of the observation in the rat model where all four regioisomers of EETs are being produced. It will also be of interest to address the contribution of rat Cyp2j3, which is a homolog of human CYP2J2 and is an epoxygenase [2]. Overall, the manuscript offers an interesting perspective on protective effect of EETs in a complex pathology. However, there are some major concerns on the details of the study design. The manuscript will give a more robust elucidation of protective effect of EETs in PAH with LIRI by refining some of the study designs and providing proper control experiments.

\section{Major points:}

1. In Methods section under Simulation of PAH with LIRI model in vitro and cell processing, all four regioisomers of EETs are soluble in organic solvent. It is not clear in that there is any vehicle control to account for the solvent effect or other controls for the treatments.

2. In Methods section, the subsection on how the extraction and measurement of plasma 11,12-DHET are conducted is missing.

3. In Results section, subsection 3.1, the statement that EETs are unstable in vivo is contradicting your hypothesis and reports from several different groups who are able to measure EETs from plasma, red blood cells, and tissues [3][4][5] [6][7][8][9][10]. 


\section{Minor points}

1. In Abstract section, there is a mistyped word on the last sentence. A letter " $\mathrm{t}$ " is missing in the “PI3K/Akt" pathway.

2. In Background section in the last paragraph, there is an extra letter " $e$ " in the sentence "... and inhibit TNF-alpha-induced apoptosis...."

3. In Methods section under Animal model interventions in the last paragraph, the word "thoracotomy" is misspelled twice.

4. In Methods section under Hemodynamic measurements, there needs to be a space between the word "exsanguination" and "via".

5. In the Methods section under TUNEL staining, the acronym "TUNEL" should be defined when it is first mentioned in the text.

6. In Figures section, the scales are not clear. In addition, the labels for comparison are very confusing. The authors might want to consider drawing lines between groups being compared.

7. In the Discussion section in the third paragraph, there is a mistyped letter for the alpha symbol.

8. In the Discussion section in the fifth paragraph, there is an extra "e" in "EDHF".

9. In Conclusions section, there is a mistyped word on the second from the last sentence. A letter " $\mathrm{t}$ " is missing in the "PI3K/Akt" pathway.

10. Throughout the text, any Latin words, such as in vitro, in vivo, in situ, need to be italicized.

\section{References}

1. `Tracy C. DeLozier, Grace E. Kissling, Sherry J. Coulter, Diana Dai, et al. (2007).Detection of Human CYP2C8, CYP2C9, and CYP2J2 in Cardiovascular Tissues. Drug Metab Dispos, vol. 35 (4), 682-688. doi:10.1124/dmd.106.012823.

2. `Darryl C. Zeldin, Julie Foley, Susan M. Goldsworthy, Molly E. Cook, et al. (1997). CYP2J Subfamily Cytochrome P450s in the Gastrointestinal Tract: Expression, Localization, and Potential Functional Significance. Mol Pharmacol, vol. 51 (6), 931-943. doi:10.1124/mol.51.6.931.

3. `Armando Karara, Shouzou Wei, David Spady, Larry Swift, et al. (1992). Arachidonic acid epoxygenase: Structural characterization and quantification of epoxyeicosatrienoates in plasma. Biochemical and Biophysical Research Communications, vol. 182 (3), 1320-1325. doi:10.1016/0006-291x(92)91877-s.

4. `Tatsuji Nakamura, Donna L. Bratton, Robert C. Murphy. (1997). Analysis of Epoxyeicosatrienoic and Monohydroxyeicosatetraenoic Acids Esterified to Phospholipids in Human Red Blood Cells by Electrospray Tandem Mass Spectrometry. J. Mass Spectrom., vol. 32 (8), 888-896. doi:10.1002/(sici)1096-9888(199708)32:8<888::aid-jms548>3.0.c0;2-w.

5. ^Houli Jiang, John Quilley, L. Manmohan Reddy, John R. Falck, et al. (2005). Red blood cells: reservoirs of cis- and trans-epoxyeicosatrienoic acids. Prostaglandins \& Other Lipid Mediators, vol. 75 (1-4), 65-78. doi:10.1016/j.prostaglandins.2004.10.003. 
6. `Sophie Goulitquer, Yvonne Dréano, François Berthou, Laurent Corcos, et al. (2008). Determination of epoxyeicosatrienoic acids in human red blood cells and plasma by GC/MS in the NICI mode. Journal of Chromatography B, vol. 876 (1), 83-88. doi:10.1016/j.jchromb.2008.10.035.

7. 'Katherine N. Theken, Robert N. Schuck, Matthew L. Edin, Bryant Tran, et al. (2012). Evaluation of cytochrome P450-derived eicosanoids in humans with stable atherosclerotic cardiovascular disease. Atherosclerosis, vol. 222 (2), 530-536. doi:10.1016/j.atherosclerosis.2012.03.022.

8. A Akinyemi Oni-Orisan, Matthew L. Edin, John Andrew Lee, Michael A. Wells, et al. (2016). Cytochrome P450-derived epoxyeicosatrienoic acids and coronary artery disease in humans: a targeted metabolomics study. Journal of Lipid Research, vol. 57 (1), 109-119. doi:10.1194/jlr.m061697.

9. `Maxwell Zeigler, Dale Whittington, Nona Sotoodehnia, Rozenn N. Lemaitre, et al. (2018). A sensitive and improved throughput UPLC-MS/MS quantitation method of total cytochrome P450 mediated arachidonic acid metabolites that can separate regio-isomers and cis/trans-EETs from human plasma. Chemistry and Physics of Lipids, vol. 216 , 162-170. doi:10.1016/j.chemphyslip.2018.09.004.

10. `Theresa Aliwarga, Xiaoyun Guo, Eric A. Evangelista, Rozenn N. Lemaitre, et al. (2020). Higher Epoxyeicosatrienoic Acids in Cardiomyocytes-Specific CYP2J2 Transgenic Mice Are Associated with Improved Myocardial Remodeling. Biomedicines, vol. 8 (6), 144. doi:10.3390/biomedicines8060144. 